$\begin{array}{ll}\text { Italique } & \text { Italique } \\ \text { Poésie italienne de la Renaissance }\end{array}$

XVII | 2014

Varia

\title{
Erotismo e sensualità nella lirica rinascimentale. Introduzione
}

Franco Tomasi

\section{(2) OpenEdition}

Journals

\section{Edizione digitale}

URL: http://journals.openedition.org/italique/381

DOI: 10.4000/italique.381

ISSN: 1663-4438

\section{Editore}

Librairie Droz

\section{Edizione cartacea}

Data di pubblicazione: 1 ottobre 2014

Paginazione: 9-17

ISBN: 978-2-600-01841-8

ISSN: 1423-3983

Notizia bibliografica digitale

Franco Tomasi, «Erotismo e sensualità nella lirica rinascimentale. Introduzione », Italique [Online], XVII | 2014, online dal 01 octobre 2016, consultato il 03 mai 2019. URL : http://

journals.openedition.org/italique/381 ; DOI : 10.4000/italique.381

(C) Tous droits réservés 


$$
\text { Fr A N C O TOM A I }
$$

E R O T I S M O E S E N S U A L I T À

NELLA LIRICA RIN A SCIMENTALE.

I N T R O D U Z I O N E 



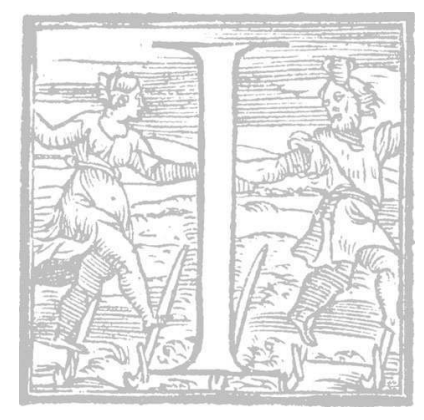

l tema dell'erotismo e della sensualità all'interno della tradizione lirica rinascimentale comprende, a ben vedere, un territorio cosi ampio e sfaccettato che è necessario, in via preliminare, perimetrare con rigore $i$ confini della ricerca. In questo senso andrà subito precisato che $i$ saggi raccolti in questo volume costituiscono un sondaggio - a più voci e da punti di vista tra loro diversi ma, si spera, convergenti - mirato a investigare la presenza del tema nel settore della cosiddetta lirica 'alta', con il che si esclude dall'indagine l'ampia tradizione comico-burlesca e talvolta francamente oscena, che pure conosce in questo periodo una ragguardevole fortuna, come documenta il precedente volume di questa rivista curato da Chiara Lastraioli. Si tratta di una scelta che nasce dal riconoscimento della sostanziale differenza costitutiva dei due generi, che vivono parallelamente e che rispondono ad istanze e a modelli significativamente diversi, tanto che per lungo tempo saranno destinati a non intersecarsi. Circoscrivere quindi al settore della lirica 'alta' lo sguardo critico significa, inevitabilmente, tornare a fare $i$ conti, sia pure in forma indiretta, con il modello che a vario titolo ne costituisce un numero primo, imprescindibile, sia quando lo si tradisca sia quando lo si faccia divenire idolo da riprendere con calligrafica precisione. Ci stiamo riferendo, ovviamente, a Petrarca e alla sua esperienza lirica, nella quale il tema della sensualità e dell'erotismo è sostanzialmente eluso, almeno nelle forme in cui la tradizione classica - specie gli elegiaci latini - lo aveva proposto; non che sia del tutto assente una pulsione erotica in senso stretto nel Canzoniere, non che manchino pagine di più aperto sensualismo, ma ciò che conta è che esse paiono confinate in un rarefatto paesaggio mentale in cui vengono 'virati' gli approdi di matrice realistica e sensuale. Vi sono, come ricorda Beatrice Bartolomeo in uno studio che costituisce un ideale prodromo alle riflessioni che hanno motivato questo volume, almeno due innegabili sbilanciamenti in direzione erotica nei Fragmenta, e si trovano inseriti, non a caso, nelle due sestine A qualunque animale alberga in terra $(\operatorname{Rvf} 22,3 I-36)$ e Non à tanti animali il mar fra l'onde (Rvf 237, 3I-36); si tratta però di un apparente sconfinamento, perché in realtà viene assai ben arginato, all'interno di un genere metrico, quello della sestina - geneticamente 
predisposto a dare ospitalità al tema della passione sensuale -, che riconduce la deriva di carattere erotico, il manifestarsi di un desio, all'interno di una tensione ottativa che di fatto lo stempera e, per cosi dire, lo contiene.

A partire quindi da questo dato, che costituisce anche, a ben vedere, un problema relativo alla costrittività quasi claustrofobica dell'inventio prescritta dal modello, si è cercato di traguardare, in una prospettiva di lungo periodo, quanto e con quali modalità la tradizione lirica abbia derogato dalle linee petrarchesche in nome della sensualità, il più delle volte istituendo un dialogo ampio e allargato con altre tradizioni, specie quella classica e, soprattutto, nella sua rimodulazione umanistica. Nel più ampio e per certi versi composito quadro delle esperienze liriche quattrocentesche, ad esempio, ricco di percorsi via via differenti $e$ nell'insieme non facilmente unificabili, appare evidente una disponibilità verso inserti che introducono, magari attraverso una facies linguistica petrarchescamente quasi ortodossa, elementi estranei e di chiara impronta erotica, tali da provocare un indugio su particolari descrittivi che dilatano e ampliano il canone petrarchesco della descriptio mulieris, permettendo l'intrusione di particolari ad alto tasso di sensualità (il seno, ad esempio, oggetto di indugi e ritagli descrittivi di significativa pregnanza, tali poi da permettere uno scivolamento all'evocazione di «secrete parti», in un crescendo di natura erotica). In questo senso, basti il rinvio al saggio di Beatrice Bartolomeo già citato, anche per la casistica dei testi esaminati; come anche, per osservazioni che vanno nella stessa direzione, i rilievi di Antonio Rossi circa gli apparati paratestuali che accompagnano le rime di Olimpo da Sassoferrato, allestiti in modo tale da esplicitare, se ce ne fosse il bisogno, il contesto fortemente allusivo e ad altro gradiente erotico di molte delle rime presenti in quei volumi (ad esempio: «imagina l'amante quando Camilla sta nuda»; "del luogo secreto imaginando»). ${ }^{3}$ $E$, del resto, non andrà dimenticato che uno dei canzonieri di maggior rilievo della stagione di secondo Quattrocento, gli Amorum libri di Matteo Maria Boiardo, affida al canto della sensualità un ruolo narrativo e tematico di centrale importanza, con una scelta assai poco abituale nella tradizione lirica volgare. Nel saggio di Tiziano Zanato che apre il nostro volume, si ripercorre l'intera parabola narrativa del liber con una specifica messa a fuoco del ruolo e del peso che in esso assume la dimensione erotica; attraverso un 
ricorso ad un lessico allusivo, in nome di una convenientia che non nega la possibilità di aperture alla sensualità, ma che piuttosto si preoccupa di irreggimentarla per darle diritto di cittadinanza in poesia, Boiardo disegna occasioni che, cariche di sapori lucreziani e catulliani, offrono una descrizione di una «amorosa zoglia» non esclusivamente mentale e paradisiaca, ma, anzi, ben terrena e prodotta dall'ebbrezza della carne. Proprio attraverso la rappresentazione della dimensione sensoriale, dalla più canonica vista, sino all'olfatto, al gusto e poi al tatto - senso, quest'ultimo, sostanzialmente interdetto da Petrarca Zanato osserva il formarsi di una fenomenologia dell'esperienza amorosa capace di comprendere e dare voce alla valenza sessuale, per la quale Boiardo chiama in causa, quale nume tutelare, anche il Boccaccio (un modello che pare soggiacere a molte delle escursioni in direzione sensuale riscontrabili nelle prove liriche di questa stagione tardo quattrocentesca). ${ }^{4}$ Fuori dal quadro qui presentato resta un'esperienza di significativa importanza, destinata a prolungarsi oltre il secondo Quattrocento con ricadute complesse e diversamente interiorizzate, vale a dire la rilettura di tutto il repertorio amoroso in una chiave filosofica sostanzialmente riconducibile, almeno in prima battuta, al pensiero ficiniano. Il peso di questa cultura di impronta neoplatonica, talvolta banalizzata e trivializzata proprio in seno alla tradizione lirica e alla trattatistica sull'amore, comporta anche la nascita di un bagaglio di strumenti esegetici per la lirica, in alcuni casi spinti anche in direzione ermetica, che spesso motivano o filtrano le esperienze di impronta sensoriale; è persino banale ricordare l'importanza che assume il fenomeno, ma è opportuno farlo almeno per giustificare il fatto che in questa sede si è preferito lasciarlo sullo sfondo, per sondare altre piste interpretative forse non sempre considerate con il giusto rilievo dalla letteratura critica. Piu in particolare, si sono volute indagare le tradizioni e le forme attraverso le quali la dimensione sensuale ed erotica coinvolge un più largo giro di dinamiche intertestuali, specie quelle che chiamano in causa la tradizione classica, anche quella esclusa da Petrarca, ma anche, e si direbbe soprattutto, la poesia neolatina di matrice umanistica, che costituisce un ricco e proficuo bacino di raccolta per temi e movenze estranee a quelle legittimate dalla tradizione volgare. In questa direzione lo studio della fortuna del tema del sogno erotico nella lirica, indagato da Erika Milburn tenendo in debito conto l'estesa parabola della sua durata, che dall'antico giunge sino al moderno, ancora una volta filtrato 
$e$ in qualche modo ridisegnato dall'esperienza petrarchesca, offre una prova di notevole significatività. In questa sorta di sottotema, apparentemente privo di uno spazio autonomo, ma spesso capace di vivere in forme parassitarie, interno cioè agli elementi più topici, la Milburn osserva come si attivi una sostanziale riapertura del rapporto con la tradizione classica, specie con gli elegiaci, a partire da Properzio, ma, in realtà, anche con una tradizione volgare extrapetrarchesca, che spesso assume le forme della poesia pastorale, e anche con un ampio spettro di testi della poesia umanistica coeva, assai più disponibile, come si è già avuto modo di osservare, alla frequentazione di temi estranei alla tradizione volgare. Proprio il rapporto tra questi due esperienze, quella umanistica e, insomma neolatina, e la produzione volgare pare essere, anche al di là degli esiti cui gli studi presentati giungono, uno dei settori di piu promettente futuro per la ricerca, in nome di una lettura che valorizzi in modo più sostanziale l'osmosi tra le due tradizioni. Non solo nei casi estremi in cui il rapporto con le lingue classiche comporta persino il tentativo di ridefinire il quadro delle istituzioni metriche come nel caso dei Versi nuovi ecc. promosso da Tolomei - ma anche il cospicuo fronte della ripresa della poesia breve ed epigrammatica, che dalla riscoperta e dal massiccio utilizgo dell'Antologia planudea apre, e spesso proprio in dimensione erotico-sensuale, a un'iniezione di tessere che non banno solo la funzione di impreziosire un dettato lirico petrarchescamente impostato, ma che finiscono, in una logica di imitazione più ampia, per provocare una torsione progressiva verso una progressiva metamorfosi del genere, tanto nei suoi istituti formali quanto nei suoi possibili esiti tematici. ${ }^{5}$ In questo senso il contatto con una tradizione, antica e moderna, di matrice classica, costituisce per molti autori una linfa capace di vivificare un linguaggio lirico sentito come sfibrato e frusto, in direzione di una difficultas che proprio allargando a una biblioteca di riferimento più ampio può essere felicemente perseguita. Si tratta di una dialettica tra tradizioni molto frequentemente riscontrabile anche nello stesso autore, una scambio che spazia dalle forme più piane del volgarizzamento alle più complesse e sfaccettate forme del rifacimento, mimando movenze stilistiche e percorsi tematici alternativi; utile sarebbe, a questo proposito, anche solo passare in rassegna le numerosissime citazioni di classici greci e latini che capita di incontrare tradotti all'interno della trattatistica o nelle lezioni accademiche di questi anni, spesso destinate a costituire preziose auctoritates 
per la discussione filosofica del tema amoroso. La traduzione quindi, intesa nel suo senso più ampio, appare un privilegiato terreno di incontro tra una lingua lirica di impostazione bembiano-petrarchesca e declinazioni tematiche extraterritoriali rispetto a Petrarca. Già nella prima metà del Cinquecento vi sono casi di sicuro interesse, magari per le forme di un rifacimento dei modelli classici che si accompagnano a un lavoro di depotenziamento dei toni troppo esplicitamente erotici allinterno della tradizione lirica. Si pensi, ad esempio, al Trissino imitatore dell'ode oraziana III 9 nel serventese Mentre che a voi non spiacqui, oppure dell'esperienza di Alamanni nel genere dell'elegia, fortemente connotata in senso tibulliano, per cio che concerne i modelli di riferimento; o, ancora, alle riscritture oraziane proposte da Alessandro Piccolomini nei suoi Cento sonetti (in particolare, il sonetto Te per tal donna, Algier, tormenta Amore, riscrittura della seconda satira del primo libro). Ma un numero di occasioni ancora più fruttuoso di confronto con il classico si riscontra anche nel settore dei volgarizzamenti o delle riprese dei poemetti di misura più lunga: una testimonianza di questo fenomeno offre il saggio di Andrea Torre, nel quale lo studioso analizza le numerose riscritture del mito ovidiano di Adone in ambito lirico, in nome della estrema variabilita degli esiti e delle possibilità che lo stesso mito offre alla tradizione rinascimentale. In un ricco intreccio di generi, che trova nelle stanze uno dei luoghi privilegiati della riscrittura rinascimentale, Torre ha modo di mettere in rilievo il diverso gioco di interpretazioni, magari attraverso il recupero di un linguaggio di impronta petrarchesca, di una situazione esplorata facendola esplodere in tutte le valenze erotiche e sensuali, generando cosi una torsione del linguaggio lirico.

Allinterno della lunga durata del tema nel corso del Rinascimento osservata in questo volume, un momento di passaggio e insieme di svolta sembra prendere forma tra gli anni Cinquanta e gli anni Sessanta del Cinquecento, quando cioè il petrarchismo, sia pure inteso come sistema o come una pluralità, sembra essere sempre più spesso, sotto la spinta di un'esigenza di adeguamento a una modernità pressante, messo in discussione: non nelle forme di una rottura frontale, ma piuttosto attraverso una metamorfosi sottile, che produce un progressivo slittamento verso un sistema di regole e di convenzioni rinnovato. E, in questa direzione, la tensione verso la dimensione sensuale ed erotica costituisce un sicuro campo di possibilità, un fronte di apertura che trova spazio 
tanto nelle esperienze collettive delle antologie - un dato messo in evidenza ancora dal saggio di Erika Milburn - quanto in quello piu studiatamente personali e mirate a dare forma compiuta a un vero e proprio liber. Il saggio di Giacomo Comiati, dedicato alla puntuale ricostruzione della parabola amorosa rinvenibile nel Canzoniere di Celio Magno, permette proprio di osservare, in una delle voci poetiche di maggior personalità del secondo Cinquecento italiano, il profondo ripensamento del libro di poesia operato dal poeta veneziano, ancora una volta non dimentico del modello petrarchesco, ma capace di rimodularlo secondo linee nuove, non prive di significativi apporti classici. Proprio in un autore che lamentava la sterilità di un esercizio lirico esclusivamente basato sul tema amoroso, ormai frusto e, alla lunga, persino svilente per il genere letterario stesso, si coglie invece una nuova messa a regime sia del tema, sia della sua operatività in seno a un'architettura tematiconarrativa. Sono soprattutto gli apporti oraziani e properziani, ma piu in generale la disponibilità ampia e larga verso una biblioteca di autori classici, dal Virgilio bucolico all'inevitabile Ovidio, che permettono a un tempo di ridare cittadinanza alle occasioni erotiche di una storia d'amore, e, insieme, le caricano di un valore altro, in nome del quale la tensione sensuale entra in dialettica con $i$ temi morali e sacri all'interno di una ambiziosa storia del sé; prende insomma forma una scrittura lirica che intende, anche attraverso le stazioni di un racconto sensuale, riformulare un racconto di matrice sostanzialmente autobiografica.

Rispetto a quanto si è osservato circa l'arco temporale che dagli anni Sessanta conduce verso il tramonto del secolo, bisognerà aggiungere nel piu ampio quadro di agenti di tensione che cooperano alla metamorfosi del genere (spinte verso la gravitas; specializzazioni tematiche dei canzonieri - sacre, encomiastiche, ecc. -; progressiva autonomia di forme, come il madrigale, strettamente connesse al rapporto con la musica; crescente peso dell'esegesi), la dimensione sensuale, che trova in Torquato Tasso uno dei suoi esponenti più brillanti, al solito, capace di illuminare tensioni e di aprire nuove strade, destinate, di li a pochi anni, a divenire poi punti di avvio che segneranno, assai probabilmente, la crisi e la definitiva rottura di un linguaggio e di una tradizione. La preferenza accordata da Tasso al tema sensuale ed erotico è ravisabile persino tra le rime tassiane di carattere encomiastico; si pensi, ad esempio, al libretto encomiastico dedicato, tra I578 e I580, "Alle Signore Principesse di Ferrara», all'interno del quale si trovano infatti pezzi 
lirici di evidente sensualità (che si soffermano, ad esempio, sul tema del seno e della fisicita). ${ }^{6}$ E il nome di Tasso ci permette di osservare anche come queste forme di lirismo siano quelle piu facilmente candidate ad essere ritrovate, quasi fatalmente, nella tradizione del poema epicocavalleresco, che del gioco di intrecci tra amori e armi aveva fatto una sua caratteristica fondante. Proprio l'esposizione stilistica in direzione sensuale costituisce il fronte piu affascinante e insieme problematico della presenza dello stile 'fiorito' all'interno della Liberata, quando all'evidente erotismo delle descrizioni si verrà aggiungendo la dimensione, per cosi dire, spettacolare e quasi teatrale che coinvolge i punti di vista di chi osserva e partecipa alle descrizioni. Basterebbe, in questo senso, tornare con la memoria alle descrizioni segretamente affini del passaggio di Sofronia tra i cittadini di Gerusalemme nel canto secondo, mentre si appresta a un sacrificio personale in nome della fede, e quella dell'arrivo di Armida nel campo cristiano nel canto quarto, anch'essa desiderosa di attirare insieme gli sguardi e di suggerire i pensieri, con un moto, quello del passaggio, ad esempio, dalla scollatura e dell'affacciarsi del seno («parte appar de le mamme acerbe e crude») all'immaginazione degli «occulti secreti» di chi la osserva, che ricorda le movenze riscontrabili nelle descriptiones mulieris quattrocentesche che abbiamo ricordato in precedenza. La storia dell'incrocio di generi e di stili, in particolare tra la lirica e il poema cavalleresco, appare del resto come uno dei terreni più fertili, tra stravolgimento parodico, sottile allusività, demistificazione di un codice espressivo, della sperimentazione in direzione sensuale di un linguaggio a dominante lirica: il caso di Ariosto, cui sarà sufficiente fare un cenno, è quanto mai palese per il rapporto stretto tra la sua propria esperienza lirica, posta sotto il segno di un marcato sensualismo, e i successivi approdi del tema nella grande narrazione del Furioso (che troveranno, anche fuori d'Italia, lettori interessati). ${ }^{7}$ Si tratta quindi di una deriva verso un sensualismo di marca lirica che conosce poi una fioritura tematica, dai baci alle più o meno dichiarate situazioni erotiche più ardite, che verrà ereditato dalla generazione successiva a quella tassiana con l'intenzione di mettere a frutto, in modo aperto e privo ormai di reticenze, un nuovo registro lirico, disponibile a una apertura tanto esibita da suscitare ben presto le attenzioni e le riserve della censura ecclesiastica. Il caso di Marino, cui è dedicato il saggio di Emilio Russo che chiude la sezione monografica di questo volume, è quanto mai indicativo di un approdo a una stagione 
in cui la metamorfosi del genere lirico, cui si accennava in precedenza, sembra essere giunta a compimento, visto il diverso rapporto con la tradizione, e, soprattutto, il definitivo sconfinamento dei territori dell'inventio di stretta osservanza petrarchesca. Come opportunamente rileva Russo, nel genere degli epitalami, talvolta cosi espliciti da indurre lo stesso poeta a occultarli o a renderli almeno non riconducibili cosi facilmente alla sua mano, Marino sperimenta un trattamento della materia lasciva che presenta forti omologie con diversi passaggi dell'Adone.

Franco Tomasi 
I. La stessa studiosa è curatrice anche di un numero monografico della rivista Seizième siècle (20 I I) dedicato al tema dell'erotismo nel Rinascimento, cui si rinvia anche per un selezionato panorama bibliografico.

2. B. Bartolomeo, Linee tematiche sensuali nella lirica di ispirazione petrarchesca del Quattrocento: alcuni esempi, in Le forme della tradizione lirica, a cura di G. Baldassarri e P. Zambon, Padova, Il Poligrafo, 20 I 2, pp. 37-60.

3. Vedi A. Rossi, Indicatori di lettura nelle «Opere» di Olimpo di Sassoferrato, in Il poeta e il suo pubblico. Lettura e commento dei testi lirici nel Cinquecento. Convegno internazionale di studi (Ginevra, I 5-I7 maggio 2008), a cura di M. Danzi e R. Leporatti, Genève, Droz, 20I 2, pp. 483-98, in part. p. 492; non sarà superfluo ricordare che libri di poesia come quello di Olimpo avrebbero goduto di una discreta fortuna anche in pieno Cinquecento presso lettori stranieri, specie in terra francese; lo stesso Rossi, del resto, ricorda come presso la British Library sia conservato un esemplare dell'edizione delle poesia di Olimpo con un ex libris di Philppe Desportes (e anche di Siméon-Guillaume de La Roque e, probabilmente, di EdouardThomas Simon de Troyes). Sugli acquisti di libri di poeti cortigiani da parte di Francesco I e sulle riprese di questa poesia nella poesia del sovrano mi sia consentito di rinviare a F. Tomasi, La poésie italienne à la cour de François I I': Alamanni, Martelli et autres cas exmplaires, in La poésie à la cour de François $I^{e r}$, sous la direction de J.-E. Girot, Paris, Pups, 201 2, pp. 65-88.

4. Cfr. Bartolomeo, Linee tematiche, cit., in part. pp. 47, 52-53 (rapporti delle rime di Giusto de' Conti e Nicolò Cosmico con il Filocolo e la Comedia).

5. Sul rapporto tra le forme brevi e la poesia italiana del XVI secolo, anche per le tangenze con il tema erotico, si veda G. Forni, Forme brevi della poesia tra Umanesimo e Rinascimento, Pisa, Pacini, 200I, in part. cap. V.

6. Sono i testi numerati, secondo l'edizione Capra (Ferrara, Corbo, I980), 2, 6, 9, ispirati a una sorprendente «audacia erotica», come sottolinea Matteo Residori nel prezioso intervento Teoria e prassi dell'encomio nel Tasso lirico, in Forme e occasioni dell'encomio tra Cinque e Seicento, a cura di D. Boillet e L. Grassi, Lucca, Pacini Fazzi, 20 I I, pp. I9-49.

7. Si veda M.C. CABAni, Le Rime e il Furioso, in Fra satire e rime ariostesche, a cura di C. Berra, Milano, Cisalpino, 2000, pp. 393-427; per l'influenza dell'Ariosto più esposto sul fronte della sensualità sui poeti francesi si veda almeno A. GENDRE, La Pléiade entre Bembo et l'Arioste, in «Italique», vI, 2003, pp. 9-36. 